Revista de

Contabilidade e

Organizações

www.rco.usp.br
DOI: http://dx.doi.org/10.11606/rco.v11i30.134372
Journal of

Accounting and

Organizations

\title{
A influência do canal de denúncia anônima na detecção de fraudes contábeis em organizações
}

The influence of anonymous reporting channel on accounting-fraud detection in organizations

Gilson Rodrigues da Silva ${ }^{\mathrm{a}}$; Rossana Guerra de Sousa ${ }^{\mathrm{b}}$

${ }^{a}$ Universidade Federal Rural de Pernambuco

${ }^{b}$ Universidade Federal da Paraiba

Palavras-chave

Canal de denúncia interna.

Fraudes contábeis.

Controle Interno.

Programa de Integridade.

Compliance.

\begin{abstract}
Resumo
Este estudo tem por objetivo analisar a influência da presença de canais de denúncias anônimos, operados internamente, na decisão dos colaboradores das organizações em comunicar casos de fraudes contábeis. Foi conduzido um experimento de campo com 191 indivíduos que atuam com a função contabilidade em empresas brasileiras com fins lucrativos, em um dos dois vínculos de trabalho 'efetivo e precário'. Os participantes foram distribuídos em dois grupos. Para o grupo de tratamento foi manipulado a presença e a forma do canal interno de denúncia anônimo. $\mathrm{O}$ experimento foi aplicado por questionário eletrônico e os resultados foram analisados com testes não paramétricos de comparações de médias. Os resultados indicam que a presença de um canal de denúncia anônimo operado internamente aumenta a intenção dos colaboradores com vinculo efetivo em relatar uma fraude contábil. Contudo a propensão dos indivíduos com vínculo precário em denunciar não é afetada.
\end{abstract}

Keywords

Anonymous Reporting Channel.

Accounting fraud.

Internal Control.

Integrity program.

Compliance.

\section{Informações do Artigo}

Recebido: 11 de julho de 2017

Aceito: 02 de setembro de 2017

\begin{abstract}
This study aims to analyze the influence of internally operated Anonymous Reporting Channels on employees' decision in communicating cases of accounting-fraud. An experiment was conducted with 191 individuals in accounting related functions from for-profit Brazilian organizations, under two types of employment contract (tenured and not tenured ones). The participants were divided into two groups. For the treatment group, 'presence' and 'type' of the internal channel of anonymous reporting was manipulated. The experiment was applied by electronic questionnaire and the results analyzed with nonparametric tests average comparisons. The results indicate that the existence of an Anonymous Reporting Channel internally operated (e.g. internal whistleblower hotline) increases tenured employee's intention in reporting the accounting-fraud. However, the propensity to expose illegal or unethical accounting practices is not affected to non-tenured employees.
\end{abstract}

Copyright (C) 2017 FEA-RP/USP. Todos os direitos reservados

\section{INTRODUÇ̃̃O}

Os impactos na economia e na sociedade causados pelas fraudes contábeis, com destaque para os casos da Enron e WorldCom, entre os anos 2000, lançam luz sobre o tema fraude e as questões relacionadas com controles e seus requisitos para eficácia no combate a esse tipo de delito. No Brasil, casos de fraudes contábeis como o do Banco Panamericano em 2010, o da Gol Linhas Áreas, reconhecido como fraude contábil pela empresa de auditoria Deloitte em 2016, e da Orthofix International em 2017 indicam que o tema ainda mantém sua relevância.

Segundo The Association of Certified Fraud Examiners - ACFE (2016), os prejuízos com atos fraudulentos, em termos globais, são estimados em torno de U\$ 6,3 bilhões anuais, representando cerca de 5\% das receitas das entidades. Estes efeitos, nas organizações e na economia, tornam preponderante o aperfeiçoamento dos mecanismos de controles, com destaque para a disseminação de programas de integridade (compliance) e de um de seus pilares o canal de denúncia (PwC, 2016), que assume relevância neste cenário, quer por questões de ordem regulatória ou por questões comportamentais. 
No aspecto regulatório, os canais de denúncia são destacados por leis como Sarbanes-Oxley Act - SOX (2002), que incentivou a criação de canais de comunicação anônima nas empresas e pelo Dodd Frank Act (2010), que acrescentou recompensas financeiras para denúncia de atos questionáveis, em relação à contabilidade, auditoria, atividades operacionais e de gestão. Os reguladores reforçam a relevância de um sistema de denúncias e de proteção contra retaliação ao denunciante para propiciar maior efetividade nas descobertas de atos fraudulentos.

Adicionalmente, a Teoria Econômica da Criminalidade (Becker, 1968) e a Teoria das Oportunidades (Cohen \& Felson, 1980) destacam a relevância do canal de denúncia como instrumento que pode atuar no comportamento do potencial fraudador, dissuadindo-o do ato e reduzindo a ocorrência de delitos.

A regulamentação dos canais de denúncia ainda é incipiente no Brasil, quanto aos aspectos institucionais e de proteção. No âmbito do mercado mobiliário está regulamentado pela Instrução CVM 509 (CVM, 2011) e no segmento financeiro pela Resolução CMN 4567/2017. Na área pública existem normas do Poder Executivo Federa ${ }^{1}$ e, em relação ao aspecto de proteção ao servidor denunciante, a Lei de Acesso à Informação (artigo 126-A Lei $\left.\mathrm{n}^{\mathrm{o}} 12.527 / 11^{2}\right)$.

O canal de denúncia se apresenta como uma das ferramentas eficazes na recepção das comunicações de atos indevidos, tanto no setor público como no setor privado, tendo corroborado com a detecção de $42 \%$ dos casos de fraudes em organizações (PwC, 2016) e sido destacado na literatura de prevenção, detecção e dissuasão de delitos e atos questionáveis nas organizações (Near \& Miceli, 1995; Kaplan \& Schultz, 2007; Near \& Miceli, 2008; Park \& Blenkinsopp, 2009; Kaplan et al., 2009; Gao et al., 2015; Johansson \& Carey, 2016). Contudo, para ser eficaz o canal de denúncia necessita de um sistema institucionalizado de recepção e proteção ao denunciante (Burket, 2000). Esta condição independe do tipo de estrutura, entidades e organismos, ou de como é operacionalizado.

A forma adotada pelas organizações para o canal de denúncia pode variar entre uma operação independente, operada por terceiros externamente, ou desenvolvida internamente, operado por equipe da organização ou por equipe externa subordinada à administração da entidade (Burkert, 2000). Contudo, quando operado internamente apresenta vantagens de preservação de informações estratégicas e atuação mais eficiente na resolução das questões relatadas.

O canal de denúncia foi a ferramenta antifraude com maior índice de implantação nas organizações (8,9\%) de 2010 a 2016 (ACFE, 2016). Nota-se uma tendência e necessidade de encorajamento aos colaboradores, em qualquer perfil ocupacional ${ }^{3}$ para que relatarem estas condutas como forma de controle. Tal importância não se reflete nas pesquisas sobre o tema, ainda incipiente sobre as motivações do comportamento de denúncia no Brasil.

A literatura brasileira reúne alguns exemplos de estudos envolvendo o tema da denúncia de fraudes contábeis no Brasil. Entre eles, Sousa et al., (2013) enfatizou as ações de denúncias internas (whistleblowing) de empregados com percepção contábil, como mecanismo de mitigação desta tipologia de fraude. Já Vasconcelos (2015) analisou como os fatores de julgamento ético, lócus de controle, clima organizacional e materialidade de delito influenciam a intenção de whistleblowing dos auditores internos no Brasil. Em linha com o tema, Lucena et al (2015) aborda a tendência dos profissionais contábeis em denunciar atos questionáveis de seus superiores e aponta para a relevância de fatores como gravidade do ato e custo pessoal como impactantes na decisão do contador de denunciar o ato.

Por fim, quanto ao canal de denúncia, Silva, Melo e Sousa (2016) analisaram se a disponibilização de um canal de denúncia anônima afetaria a intenção dos profissionais em comunicar problemas de compliance no cenário brasileiro. Os resultados indicaram a não interferência da disponibilização do canal na intenção de denúncia dos participantes de modo geral e nem quando segmentados por área de atuação pública e privada.

Apesar da literatura internacional mostrar o efeito do tipo de canal de denúncia na propensão à denúncia pelos colaboradores da organização (ex. Brennan \& Kelly, 2007; Kaplan et al., 2008; e Ahmad, Smith \& Ismail, 2010), não se tem notícia de estudos similares no Brasil A pesquisa em Contabilidade tem se apresentado ainda restrita ao buscar métodos que indiquem ou previnam condutas antiéticas que levem a ocorrência de fraudes (Curtis \& Taylor, 2009).

\footnotetext{
${ }^{1}$ Altera a Instrução Normativa Conjunta do Ministério Público e Controladoria Geral da União (01/2016 - CGU/MP), e Lei de Responsabilização das Estatais (13.303/16).
}

${ }^{2}$ Altera o Estatuto dos Servidores Públicos Civis da União (Lei no 8.112/90).

${ }^{3} \mathrm{O}$ termo perfil ocupacional será utilizado a partir deste ponto para designar os diferentes tipos de vínculos existentes entre os colaboradores de uma organização, quer sejam vínculos efetivos, para os casos dos empregados (referenciados como profissionais) ou precários, sendo aqueles que estão presentes na organização, interagem no seu ambiente, mas não estão ligados a ela em definitivo, podendo ser terceirizados ou estagiários (para este estudo foi considerado unicamente o vínculo de estagiário). 
Dadas soluções adotadas no mercado baseada em denúncias de colaboradores, a pesquisa poderia identificar os fatores que motivam tal comportamento de denúncia, e se diferentes perfis ocupacionais apesentam comportamento diverso, fatos estes, ainda não presentes nos estudos empíricos no Brasil. Assim, propomos a seguinte problemática de pesquisa: Qual a influência da presença de canais de denúncias anônimos operados internamente nas organizações na decisão dos colaboradores de comunicar casos de fraudes contábeis?

A partir da hipótese que a decisão em comunicar atos irregulares, é afetada pelos canais de comunicação disponíveis (Near \& Miceli, 1995), e que um canal operado internamente, motivaria uma intenção maior de denúncia (Kaplan et al., 2009), este estudo tem por objetivo analisar o impacto da presença de canais de denúncias anônimos operados internamente na decisão dos colaboradores de comunicar casos de fraudes contábeis e, de forma complementar, se o perfil ocupacional do colaborador tem impacto nesta decisão.

Esta pesquisa contribui com a ampliação do referencial empírico do Brasil quanto às questões envolvidas no comportamento do denunciante, para as discussões sobre o tema do controle, com ênfase no incentivo à denúncia, bem como para o referencial conceitual na área contábil quanto aos fatores motivadores da comunicação de fraudes contábeis pelos profissionais nas organizações. Ainda, metodologicamente com a utilização de processos experimentais de pesquisa neste campo de estudo.

Esse estudo apresenta além desta introdução, uma revisão da literatura sobre fraudes contábeis e canais de denúncia, na seção 2. Na sequência são apresentados os procedimentos metodológicos envolvidos na aplicação o experimento, na seção 3, e os resultados são apresentados e discutidos na seção 4 . A seção 5 apresenta as considerações finais do estudo.

\section{REVISÃO DA LITERATURA}

\subsection{Fraudes Contábeis em Organizações}

As fraudes corporativas têm ocorrido com maior frequência na conjuntura contemporânea do ambiente empresarial competitivo, tendo como um dos mecanismos a manipulação de informação que pode causar impacto no desempenho da organização (Wells, 2008). Deste modo, empresas têm enfrentado o crescente número de casos de fraudes, levando impactos significativos à reputação e perdas financeiras, que são estimadas em $5 \%$ da receita bruta anual da entidade (ACFE, 2016).

A fraude é um ato doloso, (ACFE, 2016) cometido de forma intencional e premeditado, visando-se tirar proveito de alguma coisa. Já o erro é uma ação não intencional, sem a finalidade de causar danos, que pode ser fruto da ignorância, da imprudência, da imperícia ou da negligência. Para Rezzae (2005), são exemplos de fraudes a manipulação de transações contábeis e documentos, erros intencionais ou omissão de informações contábeis para preparação de relatórios, não aplicação intencional dos princípios contábeis, usabilidade de contabilidade no gerenciamento de lucros de forma agressiva e omissão de essência dos eventos contábeis, dentre outros.

AACFE (2016) descreve que a fraude é um fenômeno sistêmico, produzido por sinergias e executado com ações coordenadas. Assim, apresenta características que antecedem ao ato fraudulento, que servem de motivação para sua ocorrência em um contexto organizacional.

Os tipos de fraudes têm relação com o ambiente organizacional, fato que torna maior o grau de dificuldade de detecção, tanto pela peculiaridade da atividade operacional quanto pelas exigências aos colaboradores (Johansson \& Carey, 2016). Dada tal característica sistêmica as organizações passaram a demandar maiores esforços para verificar a ocorrência de fraudes, bem como formas de controle. Esse movimento se intensificou nos anos 2000 com um crescente número de empresas (Enron, WorldCom, Tyco Internacional, Xerox, Bristol Meyers Squibb, Sadia, Aracruz, Banco Panamericano, Petrobrás, dentre outros), que divulgaram demonstrações fraudulentas encobrindo sua real situação financeira.

A fraude ocupacional ocorre por ações coordenadas que procedem do uso de uma ocupação (posição na organização) para extrair benefício e enriquecimento pessoal, seja pela má apropriação de bens ou ativos da organização, seja pela aplicação deliberada de tais bens (Rezaee, 2005). A $A C F E$ (2016) desenvolveu uma das maiores contribuições na distinção dos tipos de fraudes ocupacionais, divulgando a árvore da fraude nos seus relatórios anuais. A estrutura apresenta 62 tipos de fraudes, divididas em três grupos: corrupção, apropriação indébita de ativos e demonstrações fraudulentas.

Estes grupos podem ser separados conceitualmente. A corrupção é um ato praticado por uma pessoa que se utiliza de cargos ou condição social para obter vantagem pessoal, mediante a entidade que ela representa (Wells, 2008). 
A apropriação indébita de ativos se constitui de uma gama de ações que envolvem transações financeiras, principalmente, de recebimentos e pagamentos, como também subtração de estoques e outros ativos da organização para benefício próprio (Wells, 2008). Já a fraude nas demonstrações financeiras pode envolver: (1) a manipulação dos registros financeiros, (2) a omissão intencional de eventos, transações, contas ou outras informações significativas a partir do qual as demonstrações financeiras são preparadas, ou (3) má aplicação dos princípios contábeis, políticas e procedimentos utilizados para medir, reconhecer, relatar e divulgar as transações comerciais (Johansson \& Carey, 2016). Entre as motivações das fraudes nas demonstrações contábeis estão a busca pelo aumento do preço das ações ou obtenção de empréstimos, distribuição de menores valores de dividendos aos acionistas ou ainda para evitar o pagamento de impostos.

\subsection{Canais de Denúncias}

O canal de denúncia tem sido apontado como um instrumento eficaz no combate a fraudes contábeis quer por questões de rapidez na detecção do fato, quer por redução dos incentivos comportamentais (Becker, 1968; Cohen \& Felson, 1980), tanto na área pública quanto privada (ACFE, 2016). De acordo com a ACFE (2016) os mecanismos de denúncia (hotlines) de fraude com maior utilização em 2016 foram linhas telefônicas (39,5\%), e-mail $(34,1 \%)$, web-site ou formulário on-line $(23,5 \%)$ e Fax $(1,5 \%)$.

Um canal de denúncia ou hotline pode ser entendido como um mecanismo ou serviço de recebimento de denúncias, e tem como principal função a conexão de diferentes atores dentro de um modelo regulatório (Williams, 1999). Os tipos de canais de comunicação da denúncia podem ser mecanismos internos ou externos (Dworkin \& Baucus, 1998), formais ou informais, e identificados ou anônimos (Park et al., 2008).

A denúncia interna pode causar menos impacto à organização em relação à denúncia externa, uma vez que a divulgação de informações no meio externo pode ser prejudicial à estratégia da entidade (Park \& Blenkinsopp, 2009). Ademais, a divulgação de informações privilegiadas para agentes fora da organização pode violar contratos realizados e ainda a publicidade de tais fatos sem explicação ou solução prévia pode trazer prejuizos à entidade.

Por estas razões, a denúncia interna dá à organização a oportunidade de lidar rapidamente com uma preocupação, sem a pressão e cobranças por explicações que vêm do ambiente externo. Além disso, ao corrigir problemas corporativos internamente as gerências podem garantir que as informações críticas permaneçam confidenciais e incentivar a responsabilidade organizacional e aprendizagem (Zhang, Chiu \& Wei, 2009). Ao contrário, na ausência de um canal de comunicação interno, os empregados que descobrem ilegalidades corporativas não divulgam as suas observações, colocando em risco a própria organização (Near \& Miceli, 1995).

Estudos sobre determinantes de intenções de denúncias não são conclusivos. O efeito de gênero na intenção de relatar informações de fraudes contábeis aos canais de comunicação anônimo, indicam desde a propensão maior de mulheres à denúncia (Kaplan et al.,2008), até a inexistência de influência de gênero, como nos casos de Ahmad, Smith e Ismail (2010) com auditores na Malásia, e Keenan (2000) em estudo com executivos em diversos países.

A constatação que a intenção em denunciar atos ilegais aumenta na presença de canais internos tem achados mais uniformes na literatura. Brennan e Kelly (2007) verificaram tal efeito para amostra de auditores trainnes na Irlanda. No mesmo sentido aponta estudo de Kaplan et al. (2008) e Ahmad, Smith e Ismail (2010). Os autores apontam que esta preferência pode ser relacionada à uma maior garantia da confiabilidade da informação (preservação do denunciante) e crença na realização de ações corretivas de irregularidades gerando benefícios não só para a organização como também à sociedade.

Para Kaplan et al, (2009), o canal de denúncia interna operado internamente pode ser administrado pelo departamento de auditoria interna, pelo departamento de recursos humanos ou por equipe contratada de profissionais específicos que reportam as denúncias à alta administração (PwC, 2016). Essa escolha tem vários benefícios: causa menos impacto à organização, uma vez que não ocorre a divulgação de informações no meio externo (Park \& Blenkinsopp, 2009), pode servir de parâmetro para solução de problemas coorporativos, aumentar a confidencialidade, os níveis de controle da entidade (Zhang, Chiu \& Wei, 2009) e a propensão de denúncia de níveis hierárquicos inferiores.

Não há nenhuma orientação regulatória detalhada sobre como administrar um canal de comunicação anônimo, no entanto, reguladores permitem que comitês de auditoria direcionem as ações relativas a políticas e procedimentos para sua operação, assim, a eficácia e a confiabilidade de um canal de comunicação anônimo podem variar dependendo de como é administrado, existindo diferenças mínimas entre eles no que diz respeito às variáveis que influenciam a decisão de denunciar (Dworkin \& Baucus, 1998). 


\section{PROCEDIMENTOS METODOLÓGICOS}

\subsection{Método}

Foi adotado como procedimento o experimento de campo, onde os respondentes foram apresentados a uma situação simulada, pelo relato de um cenário. A variável independente foi manipulada em um grupo experimental específico, conforme proposto por Barlow e Hersen (1984), e mantido um grupo denominado de controle, sem a manipulação da referida variável.

O desenho do experimento investiga a influência da existência ou não de canais de denúncias (anônimos e operados internamente - variável independente X) na propensão de comunicar casos de fraudes contábeis (variável dependente $\mathrm{Y}$ ). $\mathrm{O}$ experimento $2 \mathrm{x} 1$ foi composto por dois grupos e um tratamento: um grupo de controle (G1) e um grupo experimental (G2). O grupo de controle no experimento (G1) não recebeu nenhum estímulo intencional e serviu de padrão para comparação. No grupo experimental (G2), ocorreu manipulação da variável independente para a medição de seus efeitos na decisão de denunciar. A decisão de comunicar o caso de fraude contábil foi medida em escala Likert de 7 pontos: 1 - Não comunicaria com certeza; 2 - Improvável que comunicasse; 3 - Pouco provável que comunicasse; 4 - Provável que comunicasse; 5 - Muito provável que comunicasse; 6 - Comunicaria com Certeza; 7 - Não tenho responsabilidade sobre isto.

Após a apresentação do cenário, o participante atribui o valor numérico na escala Likert para sua intenção de denúncia. O item 7 foi incluso na escala Likert com a finalidade de captar as respostas dos participantes que não se sentem responsáveis por comunicar o caso de fraude, ou seja, são indiferentes à situação.

\subsection{Cenário}

A modelagem do cenário utilizado considerou os elementos de maior ocorrência de fraude contábil, como esquema de manipulação de demonstrações contábeis (lançamento sem documentos), o qual é um método comum de fraude (Kaplan et al., 2009; ACFE, 2014). O cenário foi construído a partir da adaptação proposta por Kaplan et al., (2009), e Ahmad, Smith e Ismail (2010). Sendo incluídos detalhes específicos do status da pessoa que comete a fraude, tipo de atividade e dimensão da organização, para ajudar os respondentes na compreensão do caso.

O cenário desenvolvido insere o respondente na atividade contábil de acompanhamento e registro de receitas contratuais de uma empresa, sendo destacado o comportamento esperado pela entidade, no sentido da cultura ética nas relações internas e externas de trabalho. O cenário relata a exposição do respondente a um caso de fraude contábil na organização, cometida por seu gerente contábil superior. Após descobrir a fraude contábil o respondente é questionado sobre sua intenção de denunciar o caso a administração superior da organização. Neste momento, no cenário do grupo experimental é inserida a informação quanto à existência na organização de um canal de denúncia anônima, operado internamente e é apresentada questão solicitando que indique a intenção de denúncia a um canal anônimo. No caso do grupo de controle tal informação sobre o canal de denúncia inexiste.

\subsection{Procedimentos de coleta e tratamento de dados}

O universo da pesquisa foi constituído por colaboradores de organizações, segmentados em dois perfis ocupacionais específicos a partir da natureza de seus vínculos com a organização, se efetivos ou precários, denominados de profissionais e estudantes, respectivamente. A amostra desta pesquisa tem caráter não probabilístico por conveniência.

O instrumento de coleta foi distribuído aleatoriamente a 2 grupos: G1 - Grupo de Controle: Sem canal de denúncia; G2 - Grupo de Experimento: Canal de denúncia operado internamente.

O tamanho da amostra mínimo de 150 respondentes foi estabelecido com base em pesquisas anteriores sobre o tema (Kaplan et al., 2009; Ahmad, Smith \& Ismail, 2010), tendo ao final um total de 191 respondentes distribuídos da seguinte forma: 95 no grupo de controle (G1) e 96 no grupo de tratamento (G2).

O instrumento de coleta utilizado foi o questionário estruturado, enviado via e-mail aos participantes, coletado entre julho e outubro de 2016. O questionário foi dividido em duas partes: (a) Exposição ao cenário de fraudes para os respondentes comunicarem sua decisão através de uma escala do tipo Likert de sete pontos; e (b) Dados demográficos: gênero, idade, tempo no atual serviço, tempo de experiência profissional e função em relação à contabilidade. Essas variáveis foram tratadas com o intuito de identificar variações não desejáveis na variável dependente (Hair Jr et al., 1998). 
O instrumento foi submetido à validação por um grupo de avaliadores composto por doutores e mestres na área de gestão e contabilidade de Instituições de Ensino Federais do Brasil, que resultou em um Coeficiente de Validade do Conteúdo (Fenker, Dieh \& Alves, 2011) de 0,920 no que diz respeito à clareza. Já a precisão atingiu o coeficiente de 0,880 .

Da amostra de 191 respondentes, foram excluídos os dados perdidos (3 casos) e as respostas que apontaram o ponto 7 da escala (não tenho responsabilidade sobre isso) no total de 2. casos. A motivação da exclusão do ponto 7 é decorrente de sua baixa representatividade, o que inviabilizou qualquer análise desta informação.

Para reforçar a validade interna do experimento, este estudo envolve uma amostra constituída por estudantes e profissionais com experiência e em exercício de atividades em organizações, com participação voluntária (Smith, 2014). Para análise da relação entre as variáveis entre os grupos, coletadas a partir de escala Likert, foi utilizado o teste não paramétrico de Mann-Whitney U para verificar a existência de diferenças significativas entre o grupo de controle (G1) e o grupo de tratamento $(\mathrm{G} 2)$, como também verificar de forma suplementar se há influência entre a composição dos grupos em seus diferentes perfis ocupacionais (estudantes e profissionais), e sua intenção de denúncia, identificando as frequências relativas através do grau de associação com a variável dependente.

\section{APRESENTAÇÃo E ANÁLISE dOS RESULTADOS}

\subsection{Perfil dos respondentes}

Nesta seção são examinados os perfis dos grupos, aleatoriamente constituídos, para verificar se tal composição pode influenciar na discussão dos resultados ou tratamento aplicado. A distribuição dos respondentes nos dois grupos (95 e 96) não afeta significativamente sua a análise e comparação, considerando o padrão do teste estatístico utilizado. Contudo fatores como o gênero, tempo de trabalho na organização, idade, e experiência na função (estudantes e profissionais) devem ser considerados.

Observa-se na Tabela 1 , que existe uma predominância de respondentes $(57,9 \%)$ do gênero masculino no grupo de controle (sem canal de denúncia - G1), e de 52,1\% de participantes do sexo feminino no grupo de tratamento (com canal de denúncia operado internamente - G2). Cabe destacar que esta diferença de gênero na formação aleatória dos grupos não pôde ser controlada.

Tabela 1. Composição dos Grupos por Gênero

\begin{tabular}{cc|c}
\hline Gênero & $\begin{array}{c}\text { Sem canal de denúncia } \\
\mathbf{G 1}(\mathbf{n = 9 5}) \\
(\mathbf{\%})\end{array}$ & $\begin{array}{c}\text { Com canal de denúncia } \\
\text { operado internamente } \\
\mathbf{G 2}(\mathbf{n = 9 6}) \\
\mathbf{( \% )}\end{array}$ \\
\hline Feminino & 42,1 & $\mathbf{5 2 , 1}$ \\
Masculino & $\mathbf{5 7 , 9}$ & 47,9 \\
$\begin{array}{c}\text { Diferença no } \\
\text { número de } \\
\text { respondentes }\end{array}$ & $\mathbf{1 5 , 8 \%}$ & $\mathbf{4 , 2 \%}$ \\
\hline Fonte: Dados da Pesquisa $(2016)$ & &
\end{tabular}

Na Tabela 2 são consideradas outras duas questões demográficas que podem influenciar na análise dos resultados deste estudo como a idade e o tempo de experiência profissional do respondente. Para Keenan (2000), a idade dos respondentes pode ser um fator propulsor a denúncia. Membros das organizações com idade mais avançada podem deter uma compreensão maior dos sistemas de controle das entidades, e, além disso, possuírem restrições mínimas para denunciar, em comparação com novos membros. Com relação ao tempo de experiência, Dworkin e Baucus (1998) afirmam que os novos funcionários podem relutar em fazer a denúncia por desconhecerem a cultura da entidade e ter pouco conhecimento sobre os canais de denúncia disponíveis na entidade. 
Tabela 2. Composição do Grupo por idade, experiência, atuação e ocupação.

\begin{tabular}{|c|c|c|c|c|}
\hline \multirow[t]{2}{*}{ VARIÁVEIS DEMOGRÁFICAS } & \multicolumn{2}{|c|}{$\begin{array}{l}\text { Sem canal de denúncia } \\
\mathbf{G 1}(\mathbf{n}=\mathbf{9 5})\end{array}$} & \multicolumn{2}{|c|}{$\begin{array}{l}\text { Com canal de denúncia operado } \\
\text { internamente } \\
\text { G2 }(\mathbf{n = 9 6 )}\end{array}$} \\
\hline & $\begin{array}{l}\text { Estudantes } \\
(\mathrm{n}=42)\end{array}$ & $\begin{array}{l}\text { Profissionais } \\
(\mathrm{n}=53)\end{array}$ & $\begin{array}{l}\text { Estudantes } \\
\quad(\mathrm{n}=38)\end{array}$ & $\begin{array}{l}\text { Profissionais } \\
\quad(\mathrm{n}=58)\end{array}$ \\
\hline Idade & $\begin{array}{l}23,19 \\
(2,61)\end{array}$ & $\begin{array}{l}32,25 \\
(5,92)\end{array}$ & $\begin{array}{l}24,16 \\
(2,84)\end{array}$ & $\begin{array}{l}34,31 \\
(5,70)\end{array}$ \\
\hline Tempo de Experiência Profissional & $\begin{array}{c}1,74 \\
(0,83)\end{array}$ & $\begin{array}{c}8,53 \\
(6.06)\end{array}$ & $\begin{array}{c}2,39 \\
(1,03)\end{array}$ & $\begin{array}{l}10,34 \\
(5,40)\end{array}$ \\
\hline
\end{tabular}

Fonte: Dados da Pesquisa (2016)

Nota: Média (desvio padrão entre parênteses).

Em todos os grupos, os profissionais se apresentam com participação mais expressiva, sendo o Grupo de controle (G1) o que apresenta menor relação entre profissionais e estudantes. Neste sentido, considerando que o vínculo precário, próprio dos estudantes, sua menor idade e vivência no ambiente empresarial, como podem ser examinados na Tabela 2, pode-se esperar, segundo estudos de Dworkin e Baucus (1998) e Keenan (2000), que a propensão à denúncia seja menor. A partir da análise da composição dos grupos nota-se que o G2 com composição de um número maior de profissionais e acúmulo de experiência nas organizações, além de conhecimento de seus controles e menores restrições à denúncia, podem apresentar uma maior propensão à denúncia.

A influência das variáveis gênero, tempo de experiência e idade na intenção de denúncia dos respondentes entre os grupos foi verificada através do teste de Mann-Whitney e Kruskal-Wallis respectivamente. Os testes não apontaram diferenças estatisticamente significativas em ambos os casos, indicando que tais fatores não provocaram influência na intenção de denúncia dos respondentes quando segmentados nos grupos.

\subsection{Relação entre a intenção de denúncia e presença do canal de denúncia anônimo}

Na Tabela 3 é evidenciada a média de intenção de denúncia do grupo de controle - G1 (Sem canal de denúncia) e grupo de tratamento - G2 (Com canal de denúncia operado internamente) para verificar a influência do canal de denúncia nesta decisão.

Tabela 3. Intenção de denúncia e presença do canal anônimo operado internamente

\begin{tabular}{ccccccccc}
\hline Intenção de Denúncia & $\mathbf{N}$ & $\begin{array}{c}\text { Média } \\
\text { (Desvio-padrão) }\end{array}$ & $\mathbf{1}$ & $\mathbf{2}$ & $\mathbf{3}$ & $\mathbf{4}$ & $\mathbf{5}$ & $\mathbf{6}$ \\
\hline $\begin{array}{c}\text { Sem canal de denúncia } \\
\text { (Grupo de Controle - G1) }\end{array}$ & 95 & $\begin{array}{c}4,78 \\
(1,41)\end{array}$ & 4,2 & 3,2 & 11,6 & 15,8 & 22,1 & 43,2 \\
$\begin{array}{c}\text { Com canal de denúncia } \\
\text { operado internamente } \\
\text { (Grupo Experimental -G2) }\end{array}$ & 96 & $\begin{array}{c}5,27 \\
(0,93)\end{array}$ & 0,00 & 0,00 & 5,2 & 17,7 & 21,9 & 55,2 \\
\hline
\end{tabular}

Fonte: Dados da Pesquisa (2016)

Nota: Indicações da Escala Likert variando de 1 - Não comunicaria com certeza a 6 - Comunicaria com certeza.

Os dados iniciais da Tabela 3 exprimem algumas motivações comportamentais dos respondentes quando da intenção de denúncia de fraudes contábeis. Na ausência de um canal de denúncia 7,4\% dos colaboradores se eximiriam da denúncia da fraude observada. Com a presença do canal a propensão a se eximir de denunciar desaparece (itens 1 e 2, G2 sem respostas). Similarmente, a indicação de certeza da denúncia (item 6) aumenta em $12 \%$ quando comparado ao grupo sem canal de denúncia. 
Estes dados podem indicar que a presença do canal de denúncia interno aumenta a confiança dos membros da organização, quer por maior proteção visualizada pela operação interna do canal, quer pela confiança na melhoria das práticas organizacionais. Independente da motivação, a sinalização de maior propensão a denúncia é clara nos dados apresentados.

Os dados detalhados na Tabela 3 refletem que a média no grupo G2, onde há presença de canal de denúncia operado internamente, foi maior $(5,27)$ que a intenção de denúncia média do grupo de controle $(4,78)$.

A Tabela 4 apresenta testes de médias para os grupos.

Tabela 4. Intenção de denúncia do Grupo de Controle (G1) e Grupo de Tratamento (G2)

\begin{tabular}{ccc}
\hline Intenção de Denúncia & $\begin{array}{c}\text { Média } \\
\text { (Sd) }\end{array}$ & $\begin{array}{c}\text { Mann- } \\
\text { Whitney U }\end{array}$ \\
\hline Sem canal de denúncia & 4,78 & \\
(Grupo de Controle - G1) & $(1,41)$ & 0,025 \\
Com canal de denúncia operado internamente & 5,27 & \\
(Grupo Experimental - G2) & $(0,93)$ &
\end{tabular}

Fonte: Dados da Pesquisa (2016).

A Tabela 4 reflete que a intenção de denúncia dos respondentes do grupo experimental (G2) com a presença do canal de comunicação anônimo operado internamente, apresenta significância estatística $(w=-2,246$; $p=0,025)$, em relação às respostas do grupo de controle. Verifica-se que a existência do canal de denúncia anônimo como recomenda a SOX (2002), CVM (2011), e Lei 13.303/16 na organização tem relação com a maior propensão de denunciar dos funcionários.

Esses dados indicam que a presença do canal de denúncia interna aumenta a probabilidade dos empregados denunciarem fraudes e que há uma relevância no formato de operação dado ao canal de denúncia pela organização na decisão de informar a fraude tomada pelo colaborador.

O resultado está em linha com os achados dos estudos de Kaplan et al. (2009), que apontam uma possibilidade maior de denúncia interna quando da presença de canais anônimos operados internamente, especialmente por encorajar o empregado a comunicar sem medo de punições e/ou represálias por parte de gestores ou de colegas de trabalho. Isso reforçaria, como em Lucena et al (2015), para os profissionais contábeis a sensação de responsabilidade em denunciar. Segundo os autores, menor medo de represálias afetaria positivamente a intenção de comunicação de atos questionáveis, gerando um ciclo de estímulos contínuos.

Observa-se uma possível influência dos perfis ocupacionais dos grupos formados na aplicação do experimento. Considera-se a diferença entre estudantes e profissionais, estes últimos com maior conhecimento e vivência no ambiente organizacional para definição dos impactos dos canais sobre sua decisão. A Tabela 5 traz as intenções de denúncias dos grupos e as análises segmentadas por perfil ocupacional.

Tabela 5. Intenção de Denúncia do Grupo de Controle e Grupo Experimental (G2) - Por Perfil Ocupacional

\begin{tabular}{ccc}
\hline $\begin{array}{c}\text { Intenção de Denúncia de Profissionais (vínculos } \\
\text { efetivos) }\end{array}$ & $\begin{array}{c}\text { Média } \\
\text { (Desvio padrão) }\end{array}$ & $\begin{array}{c}\text { Mann- } \\
\text { Whitney U }\end{array}$ \\
\hline Sem canal de denúncia & 4,79 & \\
(Grupo de Controle - G1) & $(1,38)$ & 0,017 \\
Com canal de denúncia operado internamente & 5,40 & \\
(Grupo Experimental - G2) & $(0,86)$ & Mann- \\
\hline Intenção de Denúncia de Estudantes (vínculos & Média \\
precários) & (Desvio padrão) & \\
\hline Sem canal de denúncia & 4,76 & 0,506 (NS) \\
(Grupo de Controle - G1) & $(1,46)$ & \\
\hline Com canal de denúncia operado internamente & 5,08 & $(1,02)$ \\
(Grupo Experimental - G2) & &
\end{tabular}

Fonte: Dados da Pesquisa (2016).

Nota: NS - não significante. 
Os resultados da significância estatística entre os dois perfis ocupacionais (efetivos e precários) indicam que no grupo que representa perfil ocupacional de vínculo efetivo com a organização há diferenças significativas $(w=-2,388 ; p=0,017)$ entre a intenção de denúncia do grupo de controle $(\mathrm{G} 1)$ e grupo experimental $(\mathrm{G} 2)$. Confirma-se a influência do canal de denúncia operado internamente na propensão de denúncia dos profissionais. Por outro lado, o grupo onde os vínculos são precários, representados pelos estudantes, aponta que a existência do canal de denúncia interno não é fator motivador para sua decisão de comunicar fraude contábil.

A não significância no grupo com perfil ocupacional de vínculo precário (estudantes) quanto ao fator da existência do canal pode ser explicada pela sua própria condição transitória na organização, que não o imputaria restrições e/ou maior comprometimento com a continuidade operacional da organização caso decida denunciar ou calar frente a conduta fraudulenta. De modo diverso, o fator de proteção, representado pela possibilidade de utilização de um canal de denúncia operado internamente no grupo participantes com vínculo efetivos, pode ser fator explicativo em sua decisão de comunicar fraude quer por terem possibilidade de continuar na entidade sem temer retaliações ou vislumbram serem beneficiados com um bom desempenho da organização no futuro.

A partir desta diferença obtida pela segmentação de grupos entre os diversos perfis ocupacionais dos colaboradores da organização, é possível melhor interpretar o resultado inicialmente apresentado pela Tabela 4. Com esta interação de análises, pode-se inferir a influência do canal de denúncia operado internamente na intenção de comunicar atos de fraude contábil, pelos colaboradores com perfil ocupacional efetivo (profissionais). Sendo coerente com sua maior experiência no mercado de trabalho, não invalidando, no entanto, a constatação obtida pelo experimento.

Este resultado está em linha com os estudos de Near e Miceli (1995); Kaplan e Schults (2007); Ayers e Kaplan (2005); Hunton e Rose (2010); e Johansson e Carrey (2016), que apontam que um canal anônimo de denúncia, como o utilizado no cenário do experimento, motivaria a intenção maior de denúncia especialmente por entender que há uma proteção contra retaliações.

Os resultados, porém, não são consistentes com os achados do estudo de Silva, Melo e Sousa (2016), na área específica de compliance no cenário brasileiro. Os achados apontam a não relevância de um canal de denúncia para os empregados brasileiros na decisão de denunciar atos irregulares ou ilegais às suas organizações. Este fato pode ser decorrente da especificidade do tipo de delito comunicado no experimento realizado pelos autores, que se referia especificamente a problemas de conformidade normativa ou regulatória, que pode ter sido entendido pelos participantes como menos propenso a retaliações ou menos relevante para o futuro da organização, e assim ter mostrado indiferença dos participantes quanto ao formato de realização da comunicação.

\section{CONSIDERAÇÕES FINAIS}

Segundo dados da ACFE (2016) as fraudes contábeis trazem os maiores prejuízos financeiros as organizações dentre as três tipologias de fraude ocupacional denotando a importância do estudo dos fatores que possam reduzir sua ocorrência.

Os resultados do estudo indicam que no cenário do Brasil a presença de um canal de denúncia anônimo e operado internamente pode influenciar na decisão do colaborador com vínculo efetivo com a organização de relatar a fraude contábil da qual tenha conhecimento. Este resultado pode ser decorrente de uma visão do colaborador quanto a importância de proteção da entidade ou de redução de sua exposição ao meio externo, como forma de manter seu vínculo funcional e ser beneficiado do crescimento ou manutenção da organização no futuro. Outros fatores podem explicar o fato do impacto da presença do canal de denúncia operado internamente na indução de uma maior propensão a denúncia. Entre eles, a crença dos colaboradores em melhorias incrementais de gestão que possam modificar os sistemas de controle existentes, com o monitoramento contínuo e auditorias. Ainda, a possível visualização de uma maior proteção a retaliações, por ventura decorrentes de suas denúncias no ambiente organizacional, o que esta alinhado com as evidências internacionais sobre o tema (Near \& Miceli, 1995; Kaplan \& Schults, 2007; Ayers \& Kaplan, 2005; Hunton \& Rose, 2010; e Johansson \& Carrey, 2016)

Apesar da recente exposição dos brasileiros ao tema da denúncia coorporativa, aos seus impactos e benefícios, fica evidenciada a maior propensão a relatar fraudes contábeis apenas quando o canal é anônimo e operado internamente. Este achado pode sugerir que uma maior disseminação entre membros da organização dos benefícios da utilização dos canais de comunicação interna como efetivo instrumento de controle possa potencializar sua intenção de denúncia e consequentemente a melhoria da efetividade dos sistemas de controles das organizações e/ou redução do potencial fraudulento, como sugerido pela Teoria Econômica da Criminalidade (Becker, 1968) e pela Teoria das Oportunidades (Cohen \& Felson, 1980). 
Os resultados podem contribuir para a reflexão de reguladores e organizações quanto à decisão de incentivar a utilização de canais internos de denúncia no Brasil, o reforço à proteção ao denunciante e a necessidade da disseminação deste instrumento de controle no seio das organizações como elemento potencialmente mitigador do risco de fraude contábil, quer por identificação e investigação rápida do delito, ou por redução da oportunidade ou aumento do risco da ação fraudulenta.

\section{REFERÊNCIAS}

Ahmad, S. A., Smith, M., \& Ismail, Z. (2010). Internal Whistle-blowing Intentions in Malaysia: Factors That Influence Internal Auditors' Decision-Making Process. In International Conference on Business and Economic Research (pp. 15-16).

ACFE. (2016). Association of Certified Fraud Examiners. Report to the nations on occupation fraud and abuse. Disponível em: <https://s3-us-west-2.amazonaws.com/acfepublic/2016-report-to-the-nations.pdf>. Acesso em 29 de maio de 2016.

Ayers, S., \& Kaplan, S. E. (2005). Wrongdoing by consultants: An examination of employees' reporting intentions. Journal of Business Ethics, 57(2), 121-137.

Barlow, B.H., \& Hersen, M. (1984). Single-case experimental design: strategies for studying behavior change. New York: Pergamon.

Becker, G. S. (1968). Crime and Punishment: An Economic Approach. Journal of Political Economy, 76 (2), $169-217$.

Brennan, N., \& Kelly, J. (2007). A study of whistleblowing among trainee auditors. The British Accounting Review, 39(1), 61-87.

Burkert, Herbert. (2000) The issue of hotlines. Protecting Our Children on the Internet: towards a new culture of responsibility. Gütersloh: Bertelsmann Foundation Publishers, p. 263-318.

Cohen, L., \& Felson, M. (1980), “Property crime rates in the United States: a macrodynamic analysis, 1947-1977”. American Journal of Sociology, 86, (1), 90-119.

CVM. (2011). Comissão De Valores Mobiliários. Disponível em: < http://www.cvm.gov.br/legislacao/inst/inst509. html>.

Curtis, M. B., \& Taylor, E. Z. (2009). Whistleblowing in public accounting: Influence of identity disclosure, situational context, and personal characteristics. Accounting and the Public Interest, 9(1), 191-220.

Dodd-Frank Act. Dodd-Frank Wall Street Reform and Consumer Protection Act . (2010) 111th United States Congress. July 21, 2010.

Dworkin, T., \& Baucus, M. S. (1998). Internal vs. external whistleblowers: A comparison of whistleblowering processes. Journal of Business Ethics, 17(12), 1281-1298.

Fenker, E. A., Diehl, C. A., \& Alves, T. W. (2011). Desenvolvimento e avaliação de instrumento de pesquisa sobre risco e custo ambiental. Revista de Contabilidade do Mestrado em Ciências Contábeis da UERJ, 16(2), 3049.

Gao, J., Greenberg, R., \& Wong-On-Wing, B. (2015). Whistleblowing intentions of lower-level employees: The effect of reporting channel, bystanders, and wrongdoer power status. Journal of Business Ethics, 126(1), 8599.

Hair Jr., J.F.; Anderson, R.E.; Tatham, R.L.; Black, W.C. (1998). Multivariate data analysis. $5^{\text {th }}$ ed. New Jersey: Prentice Hall.

Hunton, J. E., \& Rose, J. M. (2011). Retracted: Effects of Anonymous Whistle-Blowing and Perceived Reputation Threats on Investigations of Whistle-Blowing Allegations by Audit Committee Members. Journal of Management Studies, 48(1), 75-98.

Johansson, E., \& Carey, P. (2016). Detecting fraud: The role of the anonymous reporting channel. Journal of Business Ethics, 139(2), 391-409. 
Kaplan, S. E., Pany, K., Samuels, J. A., \& Zhang, J. (2009). An examination of the effects of procedural safeguards on intentions to anonymously report fraud. Auditing: A Journal of Practice \& Theory, 28(2), 273-288.

Kaplan, S. E., Pany, K., Samuels, J., \& Zhang, J. (2008). An examination of the association between gender and reporting intentions for fraudulent financial reporting. Journal of Business Ethics, 87(1), 15-30.

Kaplan, S. E., \& Schultz, J. J. (2007). Intentions to report questionable acts: An examination of the influence of anonymous reporting channel, internal audit quality, and setting. Journal of Business Ethics, 71(2), 109-124.

Keenan, J. P. (2000). Blowing the whistle on less serious forms of fraud: A study of executives and managers. Employee Responsibilities and Rights Journal, 12(4), 199-217.

Lucena, E. R.F.C.V; Melo, C.L.L; Lustosa, P.R.B; Silva, C.A.T.( 2015) Ética: a tendência de os profissionais contábeis denunciarem atos questionáveis. Revista de Contabilidade e Organizações, 9 (24), 3-17

Near, J. P., \& Miceli, M. P. (1995). Whistle-blowing: Myth and reality. Journal of management, 22(3), 507-526.

Near, J. P., \& Miceli, M. P. (2008). Wrongdoing, Whistle-Blowing, and Retaliation in the US Government What Have Researchers Learned From the Merit Systems Protection Board (MSPB) Survey Results?. Review of Public Personnel Administration, 28(3), 263-281.

Park, H., \& Blenkinsopp, J. (2009). Whistleblowing as planned behavior-A survey of South Korean police officers. Journal of Business Ethics, 85(4), 545-556.

Park, H., Blenkinsopp, J., Oktem, M. K., \& Omurgonulsen, U. (2008). Cultural orientation and attitudes toward different forms of whistleblowing: A comparison of South Korea, Turkey, and the UK. Journal of Business Ethics, 82(4), 929-939.

PWC. (2016). PriceWaterhouseCoopers. Adjusting the Lens on Economic Crime Preparation brings opportunity back into focus Economic crime: people, culture and controls. <http://www.pwc.com/gx/en/services/ advisory/consulting/forensics/economiccrimesurvey.html>; Acesso em: 27 de maio de 2016.

Rezaee, Z. (2005). Causes, consequences, and deterence of financial statement fraud. Critical Perspectives on Accounting, 16(3), 277-298.

Silva, G. R., Melo, H. P. A, \& Sousa, R. G. (2016). A Proteção do Anonimato e a Eficácia do Compliance: Um Estudo Experimental Sobre a Influência do Canal de Denúncia Anônima na Comunicação de Problemas de Compliance no Brasil In: XVI USP International Conference in Accounting, São Paulo.

Smith, M. (2014). Research methods in accounting. Sage.

Sousa, R. G., Vasconcelos, A. F., Antunes, G. A., \& da Silva, J. D. G. (2013). Fraudes contábeis e whistleblowing: uma pesquisa empírica sobre a influência da percepção contábil do delatante. Revista Universo Contábil, 9(3), 128-142.

Vasconcelos, A. F. D. (2015). Influência do julgamento ético, lócus de controle, clima ético organizacional e materialidade do delito sobre as intenções de Whistleblowing dos auditores internos no Brasil. 153f, Tese de Doutorado do Programa Multiinstitucional e Inter-Regional de Pós-Graduação em Ciências Contábeis (UFPB/UNB/UFRN), João Pessoa/PB.

Wells, J.T. (2008). Principles of Fraud Examination. 2a . Ed. John Wiley \& Sons, INC, Hoboken, New Jersey.

Williams, Nigel. (1999). The Contribution Of Hotlines To Combating Child Pornography On The Internet. Recuperado em 21 de outubro, 2015 de http:/www.Childnet-Int.Org/Downloads/Combating Child Pornography.Pdf.

Zhang, J., Chiu, R., \& Wei, L. (2009). Decision-making process of internal whistleblowing behavior in China: Empirical evidence and implications. Journal of Business Ethics, 88, 25-41. 